46 | 2011

Accueil et formation des enfants étrangers en France de la fin du XIX $X^{\mathrm{e}}$ siècle au début de la Deuxième Guerre mondiale

\title{
Les écoles françaises au Liban 1909-1943 : lieux de la « mission civilisatrice »?
}

\section{Esther Moeller}

\section{(2) OpenEdition \\ Journals}

\section{Édition électronique}

URL: https://journals.openedition.org/dhfles/2130

DOI : $10.4000 /$ dhfles. 2130

ISSN : 2221-4038

\section{Éditeur}

Société Internationale pour l'Histoire du Français Langue Étrangère ou Seconde

\section{Édition imprimée}

Date de publication : 1 janvier 2011

Pagination : 181-192

ISSN : 0992-7654

Référence électronique

Esther Moeller, «Les écoles françaises au Liban 1909-1943 : lieux de la « mission civilisatrice »?», Documents pour l'histoire du français langue étrangère ou seconde [En ligne], 46 | 2011, mis en ligne le 12 juillet 2012, consulté le 31 mai 2021. URL : http://journals.openedition.org/dhfles/2130 ; DOI : https:// doi.org/10.4000/dhfles. 2130

Ce document a été généré automatiquement le 31 mai 2021.

(c) SIHFLES 


\title{
Les écoles françaises au Liban 1909-1943 : lieux de la « mission civilisatrice »?
}

\author{
Esther Moeller
}

1 L'idée centrale de cette thèse, qui a été soutenue à l'université Jacobs de Brême (Jacobs University, Bremen) en Allemagne en janvier 2011, est que les écoles françaises au Liban $^{1}$ dans la première moitié du vingtième siècle ont d'un côté servi d'instruments de diffusion de la langue et de la culture françaises, en accord avec les intérêts politiques extérieurs des gouvernements français successifs, et de l'autre d'outils d'affirmation sociale, religieuse, économique et politique de leur clientèle libanaise. Afin de rendre compte de la diversité des orientations des écoles et de leurs clientèles, le travail prend en compte les écoles françaises catholiques, juives, protestantes et laïques qui ont œuvré au Liban.

\section{Plan}

2 Après une brève présentation du contexte historique et de l'état actuel de la recherche, sont examinésl'approche théorique et méthodologique, les sources, l'hypothèse et les arguments, ainsi que la structure de la thèse et la perspective défendue.

\section{Contexte historique}

3 L'étude s'inscrit dans le contexte historique, à la fois de l'influence grandissante des puissances occidentales au Moyen-Orient, du développement d'une politique culturelle et scolaire extérieures sous la Troisième République française et de la constitution de nations arabes après le démantèlement de l'empire ottoman. À la fin du XIX siècle, le Moyen-Orient était une région particulièrement importante, du point de vue géostratégique, et comme foyer des religions monothéistes, en particulier la religion chrétienne. Tandis que les États-Unis étaient encore réticents à s'engager dans cette 
partie du monde, l'Italie et l'Allemagne s'y activaient pour gagner et garder une certaine influence, mais les antagonistes principaux étaient constitués par la GrandeBretagne et la France. L'occupation de l'Égypte par la Grande-Bretagne en 1882 constitua un coup dur pour la France qui avait elle-même réclamé cette région. Par la suite et pour compenser cette perte, la France intensifia ses contacts avec le Liban où elle entretenait des relations de longue date avec la population chrétienne. La première fois que la France s'engagea réellement dans cette région, ce fut en 1860, lorsque l'armée française intervint dans les conflits libanais internes et que le gouvernement français soutint vivement la constitution d'une province relativement autonome vis-àvis de la Sublime Porte, portant le nom de Mont Liban/Jabal Lubnan, avec un gouverneur chrétien et une population chrétienne importante. Pour cette population chrétienne et en particulier pour la majorité maronite, unie avec Rome, en son sein, le contact étroit avec la France, fut ressenti comme bénéfique, tandis que la plupart des musulmans voyaient l'influence française comme négative, ce qui se manifesta surtout au niveau des écoles françaises, toutes catholiques en ce temps : pour les chrétiens libanais, ces écoles renforçaient leur foi, alors que les musulmans craignaient une aliénation religieuse de leurs enfants. La révolution dite jeune-turque en 1909 apporta aussi un nouveau vali (gouverneur) à Beyrouth, mais au Mont Liban la situation resta quasiment la même. Les idées modernistes et laïques qui préoccupaient le comité pour le progrès et l'unité se disséminaient aussi au Liban, en particulier à Beyrouth, où des intellectuels de toutes les confessions y souscrivaient. En France, la séparation de l'Église et de l'État de l'an 1905 avait poussé les idées lä̈ques en avant, en particulier dans le domaine de l'enseignement. La politique culturelle extérieure de la Troisième République futégalement touchée, car même si les écoles catholiques françaises continuèrent à recevoir la grande majorité des subventions du gouvernement, celui-ci soutint aussi des institutions juives de l'Alliance israélite universelle et les écoles de la Mission laïque française dans différentes régions du monde, dont le Moyen-Orient. La Première Guerre mondiale et la fin de l'empire ottoman transformèrent les influences occidentales indirectes en contrôle direct, justifié par le régime de mandat attribué à la France pour le Liban et la Syrie, et à la Grande-Bretagne pour l'Irak, la Palestine et la Transjordanie, territoires qui furent en même temps constitués en tant qu'États. Bien que la tâche de l'Étatfrançaisétait d'accompagner ces pays vers l'indépendance, la France établit de fait un régime quasi-colonial au Liban et en Syrie. Alors que les chrétiens libanais, et en particulier les maronites, avaient au début désiré ce mandat français, rejeté dès le départ par les autres communautés libanaises et syriennes, le mécontentement vis-à-vis de ce régime s'accrut. Il renforça les tendances nationalistes, présentes dans la région depuis le XIX ${ }^{e}$ siècle et mena à la création de différents partis nationalistes, réclamant soit l'union de tous les pays arabes, soit l'union de la Syrie et du Liban. Au Liban, un nationalisme particulier se développa, le "libanisme", soulignant la singularité de l'identité libanaise à part des autres pays arabes. Le rejet du mandat français, la pression extérieure venant de la Société des Nations et, pendant la Deuxième Guerre mondiale, des Britanniques, obligea les Français àaccorder l'indépendance au Liban en 1943, bien que les troupes françaises et britanniques n'aient quitté la région qu'en 1946. 


\section{État de la recherche}

4 Le sujet des écoles françaises dans le monde arabe, et plus globalement dans des régions non européennes et sous domination plus ou moins directe des puissances européennes, a suscité l'attention particulière des chercheurs ces dernières années. Cet intérêt s'inscrit entre autres dans le cadre de l'attention portée à l'enseignement dans le contexte colonial. La recherche actuelle insiste sur l'ambivalence de toute éducation coloniale entre suppression et moyen d'affirmation d'identités politiques, religieuses ou culturelles, comme le montrent parmi d'autres les travaux de Harald Fischer-Tiné sur l'Inde, de J.P. Daughton sur Madagascar, Tahiti et l'Indochine et de Nicola Cooper sur l'Indochine.

5 Dans ce contexte, l'histoire de l'idée de la «mission civilisatrice " a reçu un accueil particulier, car elle souligne le caractère ambivalent de l'enseignement colonial entre discours émancipateur et pratique hégémonique. Jürgen Osterhammel a étudié l'idée de «mission civilisatrice » comme un phénomène global aux $\mathrm{XIX}^{\mathrm{e}}$ et $\mathrm{XX}^{\mathrm{e}}$ siècles, tandis que Dino Costantini, Nicolas Bancel, Pascale Blanchard et Françoise Vergès ont mis en avant le caractère spécifique de la "mission civilisatrice française", dû à son importance comme fondement idéologique de la Troisième République. Les tensions que le dualisme entre religion et laïcité a créées, non seulement dans l'enseignement métropolitain, mais aussi dans le monde arabe, ont été soulignées par les travaux de Fanny Colonna sur l'Algérie, de Frédéric Abécassis sur l'Égypte, de Karène SummererSanchez et de Dominique Trimbur sur la Palestine, de Jérôme Bocquet et de Randi Deguilhem sur la Syrie. Alors que ces auteurs comme beaucoup d'autres ont traité des écoles françaises, pour la plus grande majorité tenues par des congrégations catholiques dans un environnement majoritairement musulman, et ont, par conséquent, mis en avant leur réception très mitigée de la part de la population, le cas du Liban diffère en raison du grand pourcentage de chrétiens. Chantal Verdeil avec ses études sur les jésuites, Jennifer Dueck avec ses travaux qui intègrent les jésuites et d'autres écoles, Nadya Sbaiti avec son étude comparative du Collège protestant français et de deux écoles libanaises, et d'autres auteurs ont su le montrer: malgré le refus des écoles françaises par certains groupes de la société libanaise, l'accueil des chrétiens leur était assuré, et leur présence ne fut que partiellement récusée une fois le régime français ressenti comme domination étrangère à la fin de son mandat. Afin de comprendre l'impact politique de la présence française, les travaux de Ellen Fleischmann, Roland Löffler et Julia Hauser sur les initiatives américaines, anglaises et allemandes d'enseignement au Liban offrent une dimension comparative importante.

6 Aucun de ces travaux n'a exploité les sources des écoles de la Mission laïque française au Liban, ni ne les a mises en relation avec les autres écoles françaises, d'obédience catholique, protestante et juive. Or, cette approche semble indispensable pour nuancer l'image de la France comme pays catholique, qui pendant longtemps perdura au Liban et dans la recherche sur ce pays. De même le focus analytique sur l'idée de «mission civilisatrice » semble apporter un éclairage nouveau qui souligne les ambivalences et la multiplicité des raisons pour lesquelles Français et Libanais ont soutenu ou rejeté les activités éducatives françaises au Liban. 


\section{Approche théorique et méthodologique}

7 La thèse se sert de différentes approches théoriques et méthodologiques afin de rendre compte des différents niveaux, de l'échelle macro à l'échelle micro, de l'interaction franco-libanaise dans le domaine de l'enseignement.

Intégrédans le contexte large des Postcolonial Studies - qui soulignent la persistance de liens coloniaux au-delà de la domination coloniale directe - l'ouvrage Orientalism d'Edward Saïd fut particulièrement important pour comprendre la complexité et la dimensionà la fois politique et culturelle des relations de dépendance entre l'Occident et l'Orient, compris comme deux catégories construites pour assurer l'hégémonie du premier sur le second. En dépit de toutes les critiques justifiées vis-à-vis de cet ouvrage, notamment pour son modèle trop simpliste, ce dernier reste néanmoins crucial pour comprendre l'impact de l'imaginaire littéraire sur la perception du Liban par les Français et la relation que ces derniers entretenaient avec leur nouvelle patrie, car tous ces éléments transparaissent à travers les sources.

9 En-dessous de cette approche plutôt théorique, l'approche de l'histoire croisée, par Michael Werner et Michel Espagne, ou des Entangled Histories par Shalini Randeria cette dernière ayant été élaborée à partir de la première pour intégrer les régions non européennes -, est très utile pour souligner l'attachement des deux sociétés et nations françaises et libanaises, pendant trop longtemps considérées comme entités distinctes, mais dont la constitution ne s'explique, en réalité, que par l'apport de l'autre, soit comme modèle à suivre ou à rejeter, soit comme justificatif politique et culturel.

Pour tenir compte enfin du niveau micro-social des acteurs concrets des écoles françaises au Liban, les deux approches d'analyse de discours et de pratiques sociales ont été utilisées. Considérer l'idée de "mission civilisatrice" à la fois comme un discours dans le sens de Michel Foucault et une pratique sociale à la suite de Pierre Bourdieu, aide à prendre en considération les aspects politiques, sociaux, culturels et religieux qui motivaient l'engagement des individus dans l'enseignement français au Liban.

\section{Sources}

11 La majorité des sources provient du côté français. À côté des archives du ministère français des Affaires étrangères, à Paris et à Nantes, et des archives du ministère de l'Instruction publique française conservées pour cette partie aux Archives nationales, les archives des différentes écoles françaises en question et de leurs sièges centraux en France et au Liban ont été consultées. Il s'agit précisément des archives de l'Alliance israélite universelle conservées à son siège à Paris, des archives de la Mission lä̈que française conservées à son siège et aux Archives nationales à Paris, des archives des CEuvres protestantes françaises à Plaisir, les archives des écoles des jésuites aux archives de la Province de France à Vanves et aux archives de la Province du ProcheOrient à Beyrouth, les archives des écoles des lazaristes aux archives de la congrégation de la Province de Paris et au collège d'Antoura au Liban, les archives des écoles des Filles de la Charité au siège provincial de la Congrégation à Beyrouth et enfin des archives des écoles des Sœurs de Saint-Joseph de l'Apparition au sein d'une des écoles à Beyrouth. 
12 En complément, les Archives nationales libanaises à Beyrouth ont été consultées en ce qui concerne la période du mandat français. Afin de prendre en considération les voix des professeurs et des élèves de l'époque eux-mêmes, des documents autobiographiques ont reçu une attention toute particulière. Finalement, des interviews ont été menées avec d'anciens enseignants et élèves des différentes écoles françaises au Liban.

\section{Hypothèses et arguments}

13 Comme évoqué plus haut, l'hypothèse principale de ce travail de recherche est que les écoles françaises au Liban étaient dominées, d'une part, par les exigences culturelles et politiques du gouvernement français qui les subventionnaient et, d'autre part, par les attentes et exigences de la population libanaise, dont la faveur leur était aussi indispensable pour pouvoir subsister. Tiraillées entre ces deux pôles qui se contredisaient dans beaucoup d'aspects, les écoles françaises, c'est-à-dire leurs administrations en France ainsi que les directeurs et professeurs sur place, essayèrent de s'adapter aux changements politiques français aussi bien que libanais, tout en maintenant leurs idées pédagogiques spécifiques. Cette constellation fit également qu'il y avait aussi bien des points en commun que des différences entre les différentes écoles françaises. Un point commun important était le sentiment de patriotisme et d'attachement des directeurs et professeurs envers la France. Même si certains d'entre eux regardaient le mandat français comme inadéquat et critiquaient son application, tous étaient persuadés de leur "mission civilisatrice » et de l'apport de la culture française à la société libanaise. L'analyse a montré que la concurrence entre elles dominait - pour la faveur et les subventions du gouvernement français, pour l'attention de la population libanaise, pour des raisons d'ordre idéologique - et que c'est seulement en temps de "crise", notamment à la fin du mandat, qu'elles coopéraient et soulignaient leurs points communs. Malgré le fait que les écoles françaises, à côté des institutions libanaises, surent instruire un nombre important d'élèves libanais (au temps du mandat français, il y avait 400 écoles françaises au Liban) et qu'elles ont aussi, bien que de façon restreinte, contribué à l'ascension sociale de certaines familles, elles se révélèrent sur un certain nombre de points insuffisantes, notamment dans la formation des enseignants, dans le domaine de l'enseignement de la langue arabe et dans celui de l'histoire libanaise. Dans ces deux derniers aspects les jésuites, qui avaient l'expérience la plus longue au Liban, se montraient les plus capables, même si leur vision de l'histoire libanaise était très particulière et minimisait le rôle de l'islam. Or, les autres institutions regardaient vers les jésuites et même la Mission laïque française, considérée comme une " ennemi intime ", se servait des manuels d'histoire libanaise de la congrégation. En ce qui concerne l'attachement des anciens élèves aussi, les écoles catholiques s'avéraient les plus couronnées de succès. Il semble que leurs anciens élèves s'y sentaient attachés par plus de liens sociaux aussi bien que religieux, que ceux des autres écoles françaises. Les écoles de la Mission laïque française enfin, sur lesquelles portait un focus particulier de ce travail, se distinguaient des autres écoles françaises surtout par leur public (seul le Collège protestant français, ouvert en 1927, lui ressemblait). Contrairement aux écoles catholiques et juives, dont la majorité de la clientèle était confessionnellement homogène, les trois communautés les plus présentes à la Mission laïque française étaient les musulmans sunnites, les grecs- 
orthodoxes et les juifs. Ce public marqua la conduite des écoles laïques et les poussa à s'éloigner du laïcisme pratiqué en métropole. À titre d'exemple, les directeurs acceptèrent les pratiques religieuses, comme le ramadan et les prières. En effet, la plupart des parents d'élèves de la Mission laïque ne partageaient pas le laïcisme militant de beaucoup de ses professeurs, mais avaient choisi l'école pour sa neutralité en matière religieuse et pour les avantages économiques et sociaux qu'elle offrait.

\section{Structure de la thèse}

14 Le premier chapitre traite de l'émergence de la notion de «mission civilisatrice » en France. Après avoir montré que cette notion était à la fois un produit de la Troisième République et de la société française, ce chapitre explique que les écoles ont pu servir de lieu privilégié pour implanter cette idée, en France aussi bien qu'à l'étranger, parce que les jeunes enfants étaient les plus aptes à la recevoir. Un troisième sous-chapitre interroge les professeurs enseignants dans les écoles françaises au Liban comme vecteur de la «mission civilisatrice » et conclut que même si beaucoup de professeurs, souvent mal préparés pour leur séjour au Liban, partageaient l'idée elle-même, la «mission civilisatrice » ne constituait pas leur motif principal, mais laissait la place à des motifs d'ordre personnel, comme le goût de l'aventure, etc.

Le deuxième chapitre, par lequel ce travail s'entame dans l'ordre chronologique, prend comme point de départ la date de l'ouverture de la première école de la Mission laïque française à Beyrouth en 1909 et examine le paysage scolaire à cette période-là. À partir de l'exemple de l'école laïque le chapitre explique qu'il fallait le soutien du gouvernement, des notables et de la population ordinaire pour ouvrir et faire marcher une école étrangère, et que le moment de la révolution jeune-turque était propice pour l'aventure laïque française, qui fut confrontée néanmoins à de nombreuses difficultés.

Le troisième chapitre se concentre sur le début du mandat et sur la prise de possession des écoles françaises par le régime mandataire, afin de répandre la culture et donc l'influence française. Le dernier sous-chapitre soulève le transfert assez important d'idées, de personnes et de pratiques éducationnelles de l'Afrique du Nord vers le Levant.

Le quatrième chapitre est dédié à l'enseignement des langues française et arabe dans les écoles. Il montre la place du français comme symbole de la langue dominante à laquelle voulaient participer les Libanais pour des raisons multiples - économiques plus souvent qu'idéologiques ou religieuses - et souligne la place négligée de la langue arabe dans la plupart des écoles françaises, à l'exception de celle des jésuites. Même la Mission laïque qui soulignait l'importance de l'enseignement de l'arabe pour satisfaire les parents musulmans et nationalistes, eut du mal à accorder une place suffisamment large à cette langue.

18 Le cinquième chapitre est centré autour de l'année 1925 et s'interroge sur le caractère plus laïque de l'enseignement français au Liban ainsi que sur la confrontation des écoles avec les différents courants nationalistes au Liban. En effet, parmi les Libanais francophiles les «libanistes » se concentrèrent autour des écoles catholiques, tandis que les nationalistes syriens et arabes envoyèrent leurs enfants à la Mission laïque française. Même si la Mission laïque reçut plus d'attention de la part du gouvernement mandataire, mieux dotée en fonctionnaires, et qui de plus voulait attirer les Libanais 
engagés dans le nationalisme arabe, cette influence reste limitée. Un sous-chapitre consacré à l'enseignement de l'histoire révèle la domination de la vision de l'histoire libanaise centrée sur les Phéniciens, telle qu'elle a été propagée par les jésuites, dans toutes les écoles françaises.

Le sixième chapitre s'intéresse aux activités extra-scolaires qui ont foisonné à partir de 1930 dans les écoles françaises au Liban. Il montre combien les écoles se sont efforcées d'établir un lien fort avec leurs élèves au-delà des cours, à travers les clubs d'anciens, le sport, le théâtre. Un autre sous-chapitre examine la participation très active des écoles françaises à l'exposition coloniale de Paris en 1931, avec une visée générale, celle de souligner leur importance au sein du régime mandataire.

Le septième chapitre qui envisage les dernières années de la période mandataire fait comprendre les efforts des écoles françaises pour s'adapter aux exigences et critiques de plus en plus ouvertes de nationalistes libanais de tous les bords. Toutes les écoles essayèrent de mettre en valeur leur contribution à la création de l'État libanais et de préparer leurs élèves à des métiers qui pourraient leur servir dans une nation en plein essor, et qui garantiraient des liens forts entre les écoles et l'élite du pays.

21 La conclusion propose un regard qui porte au-delà de l'année 1943. Elle fait aussi le constat des nombreuses permanences dans l'enseignement au Liban du temps du mandat, qui empêchaient en définitive le système d'évoluer vers la satisfaction des besoins politiques, sociaux, économiques et culturels d'une nation, d'une société, ainsi que vers son unité.

\section{Publication}

22 La publication de la thèse en allemand est envisagée pour début 2012 avec la maison d'édition Vandenhoeck et Ruprecht à Göttingen (Allemagne). Une traduction et une publication du travail en langue française sont prévues.

\section{BIBLIOGRAPHIE}

ABÉCASSIS, Frédéric (2000). L'enseignement étranger en Égypte et les élites locales 1920-1960. Francophonie et identités nationales. Thèse, université d'Aix-Marseille I.

BANCEL, Nicolas, BLANCHARD, Pascal \& VERGÈS, Françoise (2003). La République coloniale. Paris : Albin Michel.

BOCQUET, Jérôme (2005). Missionnaires français en terre d'Islam : Damas (1860-1914). Paris : Les Indes Savantes.

- (2008). La France, l'Église et le Baas. Un siècle de présence française en Syrie (de 1918 à nos jours). Paris : Les Indes Savantes.

BOURDIEU, Pierre \& PASSERON, Jean-Claude (1970). La repro-duction. Éléments pour une théorie du système de l'enseignement. Paris : Éditions de Minuit. 
COLONNA, Fanny (1997). « Educating Conformity in French Colonial Algeria », in Frederick Cooper \& Anna Laura Stoler (ed.), Tensions of Empire. Colonial Cultures in a Bourgeois World. Berkeley : University of California Press, p. 346-370.

COOPER, Nicola (2001). France in Indochina. Colonial Encounters. Oxford : Berg.

COSTANTINI, Dino (2004). Mission civilisatrice. Le rôle de l'histoire coloniale dans la construction de l'identité politique française. Paris : La Découverte.

DAUGHTON, J.P. (2006). An Empire Divided: Religion, Republi-canism, and the Making of French Colonialism, 1880-1914. New York : Oxford University Press.

DEGUILHEM, Randi (2006). « Exporter la France laïque dans la Méditerranée ottomane », in Patrick Cabanel (éd.), Une France en Méditerranée. Écoles, langue et culture françaises, XIX ${ }^{\mathrm{e}}-\mathrm{XX}^{\mathrm{e}}$ siècles. Paris : Créaphis, p. 179-190.

DUECK, Jennifer (2010). The Claims of Culture at Empire's End. Syria and Lebanon under French Rule. Oxford : Oxford University Press.

FISCHER-TINÉ, Harald (2003). « Vom Wissen zur Macht. Koloniale und ,nationale’ Bildungsmodelle in Britisch Indien, ca. 1781-1920 », in Karin Preisendanz \& Dietmar Rothermund (ed.), Südasien in der „Neuzeit“. Geschichte und Gesellschaft, 1500-2000. Vienne : Promedia, p. 90-109.

FLEISCHMANN, Ellen (2010), « Lost in Translation. Home Economics and the Sidon Girls' School of Lebanon, c. 1924-1932 », in Social Sciences and Missions, 23, p. 32-62.

FOUCAULT, Michel (1975). Surveiller et punir. Naissance de la prison. Paris : Gallimard.

HAUSER, Julia (2008). «"... das hier so furchtbar verwahrloste weibliche Geschlecht aus dem Stande heben zu helfen". Der emanzipatorische Auftrag Kaiserswerther Diakonissen im Osmanischen Reich und seine Ambivalenzen », in Wolfgang Gippert \& Petra Götte (ed.), Transkulturalität. Gender- und bildungs-historische Perspektiven. Bielefeld : Transcript-Verl., p. 219-236.

LÖFFLER, Roland (2008). Protestanten in Palästina. Religionspolitik, Sozialer Protestantismus und Mission in den deutschen evangelischen und anglikanischen Institutionen des Heiligen Landes 1917-1939. Stuttgart : Kohlhammer.

OSTERHAMMEL, Jürgen (2006). Europe, the „West” and the Civilizing Mission. The 2005 Annual Lecture. German Historical Institute London.

RANDERIA, Shalini \& CONRAD, Sebastian (2002). « Geteilte Geschichte - Europa in einer postkolonialen Welt ", in Shalini Randeria \& Sebastian Conrad (ed.), Jenseits des Eurozentrismus. Postkoloniale Perspektiven in den Geschichts- und Kulturwissen-schaften. Francfort / New York : Campus Verlag, p. 9-49.

SANCHEZ-SUMMERER, Karène (2006). « Langue(s) et religion(s) en Palestine mandataire au sein d'institutions éducatives catholiques. Établissements des Frères des Écoles chrétiennes et Sœurs de Saint Joseph de l'Apparition (1922-1940) », in Documents pour l'histoire du Français langue étrangère ou seconde, 37, p. 93-132.

SBAITI, Nadya (2008). Lessons in History: Education and the Formation of National Society in Beirut, Lebanon, 1920s-1960s. Thèse, Georgetown University.

TRIMBUR, Dominique (2006). « La revanche des congrégations ? Politique anticléricale et présence catholique française en Palestine au début du XX $\mathrm{X}^{\mathrm{e}}$ siècle », in Jean-Pierre Luizard (éd.), 
Le choc colonial et l'islam. Les politiques religieuses des puissances coloniales en terre d'islam. Paris : La Découverte, p. 121-135.

VERDEIL, Chantal (2003). Les jésuites de Syrie (1830-1864). Une mission auprès des chrétiens d'Orient au début des réformes ottomanes. Thèse, université Paris IV.

\section{NOTES}

1. Il importe de souligner que juridiquement le terme Liban ne peut être appliqué qu'à partir de 1920, date de la création de l'État libanais sous l'égide française dans ses dimensions actuelles. Le terme sera néanmoins utilisé pour la période antérieure, désignant la province du Mont Liban/ Jabal Lubnan, les villes côtières Beyrouth, Tripoli et Saida ainsi que les quatre anciens cazas ottomans de Hasbaya, Rashaya, Baalbek et Akkar.

\section{AUTEUR}

\section{ESTHER MOELLER}

Institut d'histoire européenne de Mayence, Allemagne

moeller@ieg-mainz.de 